Draft Version May 15, 2018

Preprint typeset using $\mathrm{LAT}_{\mathrm{E}} \mathrm{X}$ style emulateapj v. 11/10/09

\title{
ASTEROSEISMOLOGY OF EVOLVED STARS WITH KEPLER: A NEW WAY TO CONSTRAIN STELLAR INTERIORS USING MODE INERTIAS
}

\author{
O. Benomar ${ }^{1,6,7}$, K. Belkacem ${ }^{2}$, T.R. Bedding ${ }^{1,6}$, D. Stello ${ }^{1,6}$, M.P. Di Mauro ${ }^{4}$, R. Ventura ${ }^{5}$, B. Mosser ${ }^{2}$, M.J.

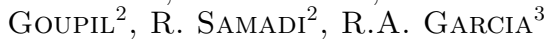 \\ Draft version May 15, 2018
}

\begin{abstract}
Asteroseismology of evolved solar-like stars is experiencing a growing interest due to the wealth of observational data from space-borne instruments such as the CoRoT and Kepler spacecraft. In particular, the recent detection of mixed modes, which probe both the innermost and uppermost layers of stars, paves the way for inferring the internal structure of stars along their evolution through the subgiant and red giant phases. Mixed modes can also place stringent constraints on the physics of such stars and on their global properties (mass, age, etc...). Here, using two Kepler stars (KIC 4351319 and KIC 6442183), we demonstrate that measurements of mixed mode characteristics allow us to estimate the mode inertias, providing a new and additional diagnostics on the mode trapping and subsequently on the internal structure of evolved stars. We however stress that the accuracy may be sensitive to non-adiabatic effects.

Subject headings: stars: oscillations, stars: interiors, methods: data analysis
\end{abstract}

\section{INTRODUCTION}

Asteroseismology, with the help of the space-borne missions such as CoRoT (e.g. Baglin et al. 2006a, b; Michel et al. 2008) and Kepler (e.g. Borucki et al. 2010) gives us access to the internal structure of stars. This is made possible through the detection of oscillation modes propagating throughout the stars (Appourchaux et al. 2008; Metcalfe et al. 2012; Gizon et al. 2013).

While for main sequence stars we observe only accoustic modes, subgiants and red giants also show a rich spectrum of so-called mixed modes (e.g. Beck et al. 2011; Bedding et al. 2011; Mosser et al. 2011a; Benomar et al. 2013). They have a dual nature, behaving as acoustic modes in the stellar envelope and as gravity modes in the core. They are therefore detectable at the stellar surface while providing information on the innermost regions. This aspect of mixed modes has motivated extensive theoretical work (e.g. Dziembowski 1971; Scuflaire 1974; Osaki 1975; Aizenman et al. 1977; Dziembowski et al. 2001; Christensen-Dalsgaard 2004; Dupret et al. 2009).

Oscillations are very sensitive diagnostics that can be used to constrain the stellar structure and to give an insight to internal properties of stars, such as the extent of the convective zone (Roxburgh 2009). In particular, mixed modes provide valuable information on the core structure which, for example, allows us to better con-

\footnotetext{
${ }^{1}$ Sydney Institute for Astronomy (SIfA), School of Physics, University of Sydney, NSW 2006, Australia

2 LESIA, Observatoire de Paris, CNRS UMR 8109, Université Paris Diderot, 5 place J. Janssen, 92195 Meudon, France

3 Laboratoire AIM, CEA/DSM-CNRS-Université Paris Diderot; IRFU/SAp, Centre de Saclay, 91191 Gif-sur-Yvette Cedex, France

4 INAF-IAPS Istituto di Astrofisica e Planetologia Spaziali Via del Fosso del Cavaliere 100, 00133, Roma, Italy

${ }^{5}$ INAF-Astrophyscial Observatory of Catania, Via S. Sofia 78, 95123, Catania, Italy

${ }^{6}$ Stellar Astrophysics Centre, Department of Physics and Astronomy, Aarhus University, Ny Munkegade 120, DK-8000 Aarhus C, Denmark

${ }^{7}$ Department of Astronomy, The University of Tokyo, 113-033, Japan
}

strain the evolutionary status of evolved low-mass stars (Bedding et al. 2011; Mosser et al. 2011a). The strength of mode mixing depends mainly on the trapping of the mode and therefore on the evanescent region between the inner and outer resonant cavities of stars.

To date, asteroseismology has generally used the mode frequencies to probe stellar interiors. In this Letter, we show that we can also use mode inertias. Theoretical calculations (e.g. Dupret et al. 2009) show that inertias of mixed modes vary with frequency in a way that is related to the efficiency of the trapping, which changes significantly as the star evolves. Moreover, Samadi et al. (2008) showed that mode inertia depends strongly on turbulent pressure. Hence, mode inertias can provide stringent constraints on mode trapping, reveal stellar interiors and potentially provide a new insight on the physics of stars. Furthermore, based on an asymptotic analysis, Goupil et al. (2013) proposed a way to observationally derive the ratio between the inertia in the $g$ cavity and the total inertia of a mixed mode.

Motivated by the latest Kepler observations, and based on the mode fitting results from Benomar et al. (2013), we show that in conjunction with frequencies, mode inertias could help us to infer precisely the cavities of oscillations. We describe in Section 2 a method for inferring mode inertias from the measurements of mode amplitudes and linewidths. In Section 3 we apply the procedure to two stars observed by Kepler, KIC 6442183 and KIC 4351319, and compare these results with models. We present diagnostics of the stellar structure of evolved stars, derived from the measurements of inertia in Section 4.

\section{INFERENCE OF MODE INERTIA RATIOS}

\subsection{Preliminary definitions}

In this section we show how to use observed mode amplitudes and linewidths to measure mode inertia for stars showing mixed modes. Let us consider a stochasticallydriven mode. If this mode is resolved, i.e. if the duration of the observations is significantly longer than the 
mode lifetime, it has a Lorentzian profile in the power spectrum (e.g. Appourchaux et al. 2012). In contrast, if the duration of the observations is shorter than the mode lifetime, the mode will have an unresolved sincsquared profile (such as expected for solar $g$ modes, see Belkacem et al. 2009). Thus, it is only possible to measure linewidths for resolved modes and we restrict our discussion to this case.

The height $H$ of a mode is given by (e.g. Libbrecht 1988; Chaplin et al. 1998; Baudin et al. 2005; Chaplin et al. 2005; Belkacem et al. 2006)

$$
H=\frac{P}{2 \eta^{2} \mathcal{M}},
$$

where $P$ is the excitation rate, $\eta$ is the damping rate, and $\mathcal{M}$ is the mode mass, which is proportional to the mode inertia $I$ (e.g. Goldreich et al. 1994):

$$
\mathcal{M}=\frac{I}{|\vec{\xi}(R)|^{2}}, \quad \text { with } \quad I=\int_{0}^{M}\left|\vec{\xi}^{2}\right| \mathrm{d} m
$$

Here, $\vec{\xi}(R), R, \mathrm{~d} m, M$ are the displacement vector, the stellar radius, the mass element and the mass of the star, respectively.

Power spectrum analysis mostly relies on fitting Lorentzian profiles to the observed modes. The usual form is

$$
L(\nu)=\frac{H}{1+4\left(\nu-\nu_{c}\right)^{2} / \Gamma^{2}},
$$

such that $\Gamma=\eta / \pi$ is the full-width-at-half-maximum (hereafter called linewidth) and $\nu_{c}$ is the central frequency. The observables needed to estimate the mode inertia are the height $H$ and the linewidth $\Gamma$. Note that they are also related to the mode amplitude:

$$
A^{2}=\pi H \Gamma / 2 \text {. }
$$

\subsection{Relation between mode inertia, amplitude and linewidth}

We recently used Kepler data to accurately measure the Lorentzian profiles of oscillation modes (Benomar et al. 2013). To go a step further, and guided by those results, we now consider the comparison of two neighboring modes:

1. a radial mode $(\ell=0)$, denoted by the subscript 0 , and

2. a dipole mode $(\ell=1)$ that exhibits a mixed character, i.e., pressure-like behavior in the envelope and gravity-like behavior in the innermost layers. The subscript 1 will be used for this mode.

We choose modes that are resolved, allowing us to apply Equation 1 for computing their heights and assume modes of neighbor frequency $\left(\nu_{0}\right.$ and $\left.\nu_{1}\right)$.

From Equation 1 and the fact that $\Gamma=\eta / \pi$, the ratio of mode heights can be written

$$
\frac{H_{0}}{H_{1}}=\left(\frac{P_{0}}{P_{1}}\right)\left(\frac{\mathcal{M}_{1}}{\mathcal{M}_{0}}\right)\left(\frac{\Gamma_{1}}{\Gamma_{0}}\right)^{2} .
$$

Note that this relation does not account for mode visibilities. These will be considered in Section 3.1
Equation (5) deserves some comments. Firstly, it links the observables $H$ and $\Gamma$ with the quantities $P$ and $\mathcal{M}$, which are only derived from modelling. Secondly, because we require the frequencies $\nu_{0}$ and $\nu_{1}$ to be close to each other (say $\left|\nu_{0}-\nu_{1}\right|<\Delta \nu / 2$ ), the shapes of the eigenfunctions in the uppermost layers are very similar. As shown by Dupret et al. (2009), this implies that the driving are the same:

$$
P_{0} \mathcal{M}_{0} \simeq P_{1} \mathcal{M}_{1}
$$

Equation 6 means that, at similar frequencies and with similar mode shapes in the super-adiabatic layers, the work done by the driving source on the modes is the same. Theoretical computations on evolved stars spanning from the early-subgiant phase to the top of the red giant branch show that this approximation is accurate to better than one percent in the observed frequency range (near $\nu_{\max }$ ) but fails at very low frequencies, where mode amplitudes are too small to be observed. Nevertheless, a thorough investigation of the accuracy of Equation 6 is desirable.

Using Equation 2 and the fact that $\left|\vec{\xi}_{\ell=0}(R)\right| \simeq$ $\left|\vec{\xi}_{\ell=1}(R)\right|$, we can consider that mode mass is proportional to mode inertia. Equation 6 can be then be recast as

$$
\frac{\mathcal{M}_{1}}{\mathcal{M}_{0}} \simeq \frac{I_{1}}{I_{0}} \simeq \sqrt{\frac{H_{0}}{H_{1}}} \frac{\Gamma_{0}}{\Gamma_{1}} .
$$

This shows that one can derive the ratio of mode inertias (or mode masses) between a radial mode and a neighboring dipole mixed-mode from the observed mode heights and linewidths. Using Equation 4 the inertia ratio can also be rewritten in terms of mode amplitudes and linewidths,

$$
\frac{I_{1}}{I_{0}} \simeq \frac{A_{0}}{A_{1}} \sqrt{\frac{\Gamma_{0}}{\Gamma_{1}}} .
$$

Note that observed height and linewidth are anticorrelated (e.g. Baudin et al. 2005), which makes it difficult to reliably measure inertia using Equation 7 when using an algorithm for which the result could be sensitive to the parametrisation (such as MLE). On the other hand, amplitudes and linewidths are weakly correlated so that using Equation 8 will provide more reliable results than Equation 7.

\section{OBSERVATIONAL DETERMINATION OF THE MODE INERTIA RATIOS \\ 3.1. Method}

Here, we show how to infer mode-inertia ratios from Kepler observations. The power spectra were prepared using methods described by García et al. (2011). We will first consider the star KIC 6442183, which was analyzed by Benomar et al. (2013) using Quarters 5 to 7 (nine months). It was selected due to its high signal-to-noise oscillation spectrum and because it does not show large rotational splittings that would result in a more complex power spectrum. Its global seismic characteristics are given in Table 1, and show KIC 6442183 to be an early subgiant star of about one solar mass.

We also considered KIC 4351319, a red giant star already analyzed by Di Mauro et al. (2011) using Q3.1 
Table 1

Main parameters for KIC 6442183 and KIC 4351319. Model A is for KIC 6442183. Model B is for KIC 4351319 and uses the same physics as model A. References: (a) Molenda-Zakowicz et al. (2013), (b) Di Mauro et al. (2011), (c) Benomar et al. (2013), (d) This study

\begin{tabular}{cc|c|c|c} 
& KIC 6442183 & Model $A^{(d)}$ & KIC 4351319 & Model $B^{(b)}$ \\
\hline$T_{\text {eff }}(\mathrm{K})$ & $5738 \pm 62^{(a)}$ & 5632 & $4700 \pm 50^{(b)}$ & 4752 \\
$\log g$ & $4.14 \pm 0.10^{(a)}$ & 4.01 & $3.30 \pm 0.10^{(b)}$ & 3.5 \\
{$[\mathrm{Fe} / \mathrm{H}]$} & $-0.120 \pm 0.050^{(a)}$ & -0.036 & $0.23 \pm 0.15^{(b)}$ & 0.20 \\
$\Delta \nu(\mu \mathrm{Hz})$ & $65.07 \pm 0.09^{(c)}$ & 65.21 & $24.38 \pm 0.1^{(d)}$ & 24.6 \\
$\nu_{\max }(\mu \mathrm{Hz})$ & $1160 \pm 4^{(c)}$ & & $380.7 \pm 4^{(d)}$ & \\
$\Delta \Pi_{1}(\mathrm{sec})$ & $325 \pm 18^{(c)}$ & 359 & $97.2^{(d)}$ & 97.8 \\
$M\left(\mathrm{M}_{\odot}\right)$ & $\sim 0.94^{(c)}$ & 1.02 & $\sim 1.26^{(d)}$ & 1.32 \\
$R\left(\mathrm{R}_{\odot}\right)$ & $\sim 1.60^{(c)}$ & 1.65 & $\sim 3.40^{(d)}$ & 3.39 \\
$V_{\ell=1}^{2}$ & 1.52 & & 1.55 & \\
\hline
\end{tabular}

data (one month). For this work, we re-analyzed the power spectrum using Q3 to Q14 data (three years) and adopting a Markov Chain Monte-Carlo sampler (e.g. Benomar et al. 2009; Handberg \& Campante 2011), allowing precise measurements of all mode parameters. Table 1 summarizes the global characteristics of this star.

Note that the measured amplitudes are not bolometric and must therefore be corrected for the Kepler spectral response. Moreover, geometric and limb darkening effects must be considered. Consequently, Equation 8 is modified to become

$$
\frac{I_{1}}{I_{0}} \simeq V_{1} \frac{A_{0}}{A_{1}} \sqrt{\frac{\Gamma_{0}}{\Gamma_{1}}},
$$

where the coefficient $V_{1}$ (hereafter called visibility) is defined as $V_{1}^{2}=H_{1}^{t h} / H_{0}^{t h}$ with $H_{0}^{t h}$ and $H_{1}^{t h}$ being theoretical heights for $\ell=0$ and $\ell=1$ modes. To compute $V_{1}$, we use the results of Ballot et al. (2011), which considered the spectral response of the Kepler spacecraft and include limb darkening and geometric effects. Ballot et al. (2011), based on the work of Michel et al. (2009), computed a grid of values for $V_{1}$ as a function of $T_{\text {eff }}, \log g$ and $[\mathrm{Fe} / \mathrm{H}]$. By interpolating this grid, we obtain the visibilities for KIC 6442183 and KIC 4351319 reported in Table 1 (see Mosser et al. 2012a, for a discussion about observed visibilities).

Using Equation [9, together with the mode linewidths and amplitudes measured from Kepler observations, it is possible to derive the mode-inertia ratios. However, one must also consider how to compute the ratio of amplitudes $A_{0} / A_{1}$ and of linewidths $\Gamma_{0} / \Gamma_{1}$. To determine these ratios, we first interpolated $A_{0}\left(\nu_{0}\right)$ and $\Gamma_{0}\left(\nu_{0}\right)$ at $\nu_{1}$ between consecutive radial modes. This relies on the fact that amplitudes and linewidths of radial modes vary smoothly from mode to mode (e.g. Christensen-Dalsgaard 2004; Dupret et al. 2009).

To robustly estimate the uncertainties, the calculation of the mode-inertia ratio involved the probability density functions of the parameters from the MCMC process. The median of the probability density function defined the most likely solution, while confidence intervals at $\pm \sigma$ were computed using the cumulative distribution function and used as the lower and upper uncertainties.

\subsection{Results}

In Fig. 11 we show the observed mode-inertia ratios for KIC 6442183 and KIC 4351319 as a function of frequency, as well as their échelle diagrams. Vertical lines are halfway between consecutive $\ell=0$ p-mode frequencies $\left(\nu_{p, 0}\right)$ and so give good estimates for the $\ell=1$ pure $\mathrm{p}$-mode frequencies $\left(\nu_{p, 1}\right)$.

For the subgiant, the mixed modes closest to the $\ell=1$ p-mode frequencies have the lowest mode-inertia ratios $\left(I_{1} / I_{0}\right.$ remains close to unity), while for mixed modes that depart significantly from the $\mathrm{p}$ modes, $I_{1} / I_{0}$ increases up to about two.

In the red giant, the density of g modes is greater than the density of the $\mathrm{p}$ modes, allowing us to better trace variations of the mode inertia. $I_{1} / I_{0}$ ranges between 1.5 and 10 because, in this regime, all modes are strongly mixed (none are pure $\mathrm{p}$ modes).

These behaviors are due to the difference in evolutionary stage. As a star evolves from subgiant to red giant, the density contrast between the core and the envelop increases, and so do the mode-inertia ratios (Dupret et al. 2009). This is also consistent with theoretical computations of mode inertias in evolved solar-like stars (e.g. Dupret et al. 2009; Montalbán et al. 2010).

\section{COMPARISON WITH STELLAR MODELS}

In this section, we show how inertias can provide new diagnostics on the internal structures of evolved stars. To this end, we first compare the observed and modeled values of $I_{1} / I_{0}$. We then discuss the potential diagnostics on the stars internal structure.

\subsection{Comparison between observed and modeled mode-inertia ratios}

The structure models of KIC 6442183 and KIC 4351319 were computed using the ASTEC evolution code (Christensen-Dalsgaard 2008b) and the associated eigenfrequencies and eigenfunctions were computed using ADIPLS oscilation code (Christensen-Dalsgaard 2008a). The input physics used for the modelling is described in detail by Di Mauro et al. (2011). The selected models well reproduce spectroscopic constraints (effective temperature and surface gravity), and the set of all the observed frequencies, as well as the large $(\Delta \nu)$ and small separations $\left(\delta_{02}\right)$. For KIC 4351319 , we used the evolutionary model previously computed by Di Mauro et al. (2011). The global parameters of the models are reported in Table 1

In Fig. 2 the ratio $I_{1} / I_{0}$ derived from the modelling is compared with observations and we see very good agreement for most modes. This strengthens the validity of 

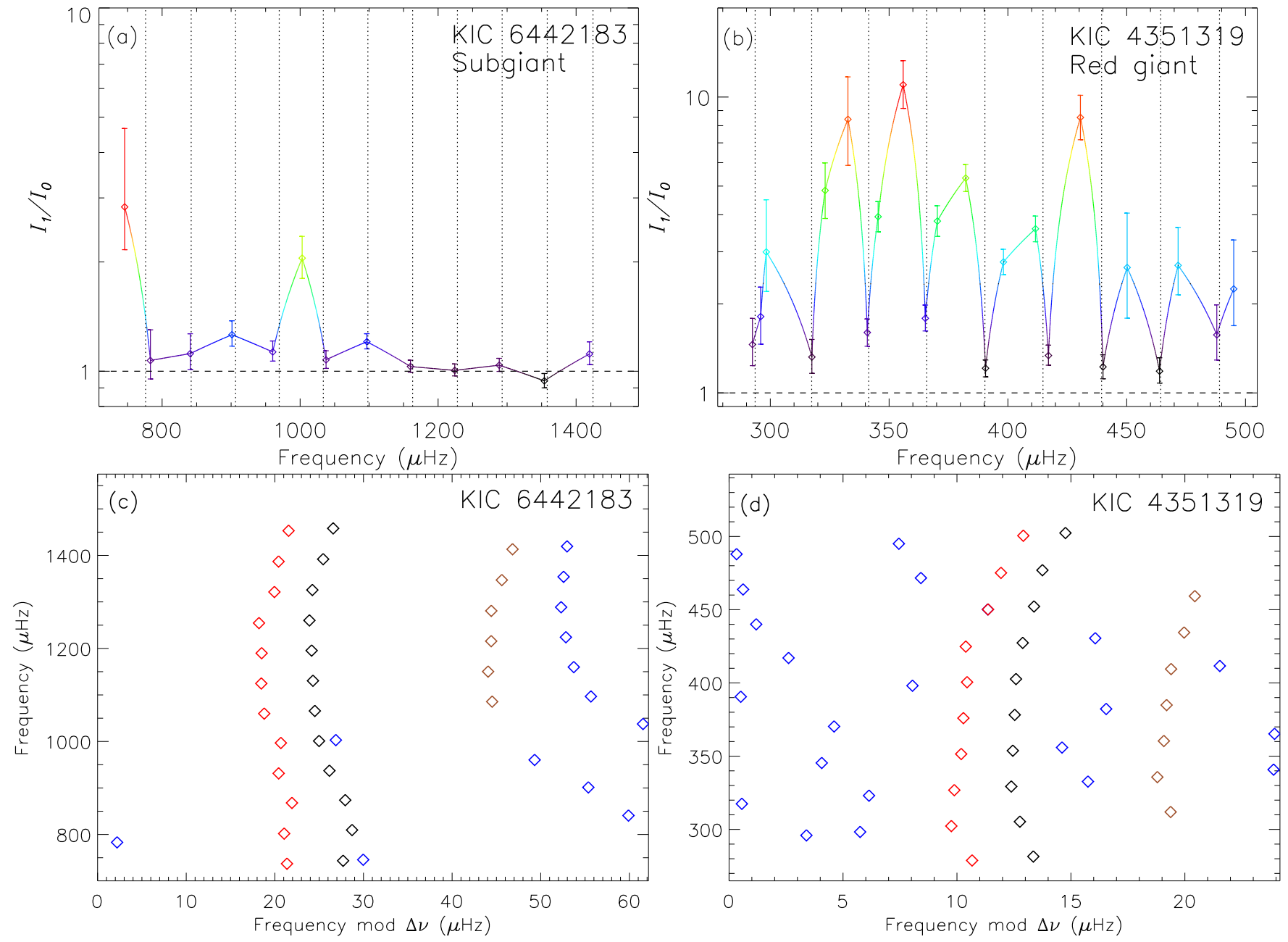

Figure 1. Inertia ratio between $\ell=1$ and $\ell=0$ modes measured for KIC 6442183 (a) and KIC 4351319 (b) against frequency. Approximate positions of the pure $\ell=1 \mathrm{p}$ modes $\left(\nu_{p, 1}\right)$ are indicated by vertical dashed lines. Échelle diagrams of observed frequencies for KIC 6442183 (c) and KIC 4351319 (d) with $\ell=0,1,2,3$ modes reported in black, blue, red, brown diamonds respectively. Warmer colors indicate high ratio, cold colors indicate lower ratio.

our method to derive the ratios $I_{1} / I_{0}$ from the observed mode amplitudes and linewidths, because the models were not chosen to reproduce the mode inertias but only to match mode frequencies. It also suggests that a more accurate model of these star could be derived using both frequencies and inertias.

\subsubsection{Small separation and period spacing diagnostics using collapsed échelle diagrams}

In the following, we focus on the red giant KIC 4351319 , since the variations in $I_{1} / I_{0}$ are greater. Échelle diagrams, either in frequency or in period help to visualise these variations and provide diagnostics on mixed modes (see Fig. 3 and Fig. 4).

Frequency-échelle diagrams are produced by representing the frequencies as a function of the difference $\nu_{1}-\left(\nu_{p, 0}+0.5 \Delta \nu\right), \Delta \nu$ being measured using the radial frequencies. Note that in contrast to a normal échelle diagram (Fig. 1 c,d), here we remove any curvature in the radial ridge by substracting the measured radial mode frequency at each order. Collapsing the échelle diagram vertically and plotting inertia on the ordinate (bottom panels) reveals clearly the variation of the inertia with frequency.

Based on the asymptotic relation for $\mathrm{p}$ mode, it is conventional to define the small separation $\delta \nu_{01}=-\nu_{p, 1}+$ $\left(\nu_{p, 0}+0.5 \Delta \nu\right)$. As shown in Fig 1] $\ell=1$ modes with the lowest inertia behave almost as pure p-modes. Therefore, the frequency position of the inertia minimum allows us to estimate $\delta \nu_{01}$. For KIC 4351319, we measured $\delta \nu_{01}=-0.69 \pm 0.30 \mu \mathrm{Hz}$ by fitting a second order polynomial within the range $[-\Delta \nu / 4, \Delta \nu / 4]$ on the collapsed échelle. For comparison, the same approach on model frequencies gives $\delta \nu_{01}=-0.53 \pm 0.24 \mu \mathrm{Hz}$ (cf. Fig 3 ). Note that negative values are found in most evolved stars (e.g. Bedding et al. 2010; Mosser et al. 2011b; Corsaro et al. 2012).

The period-échelle diagram (Fig 4) presents the frequency ratios as a function of the period $\nu_{1}^{-1} \bmod \Delta \Pi_{1}$, where $\Delta \Pi_{1}$ is the average period spacing of the $\ell=1$ g-mode frequencies (Bedding et al. 2011) . The maxima of mode inertias are separated by $\Delta \Pi_{1}$ in the periodéchelle diagram, so by collapsing vertically over the correct value of the period-spacing, the mode-inertia-period plot shows a repetitive pattern of peaks, spaced by $\Delta \Pi_{1}$ (the pattern period). This is therefore a new method to 

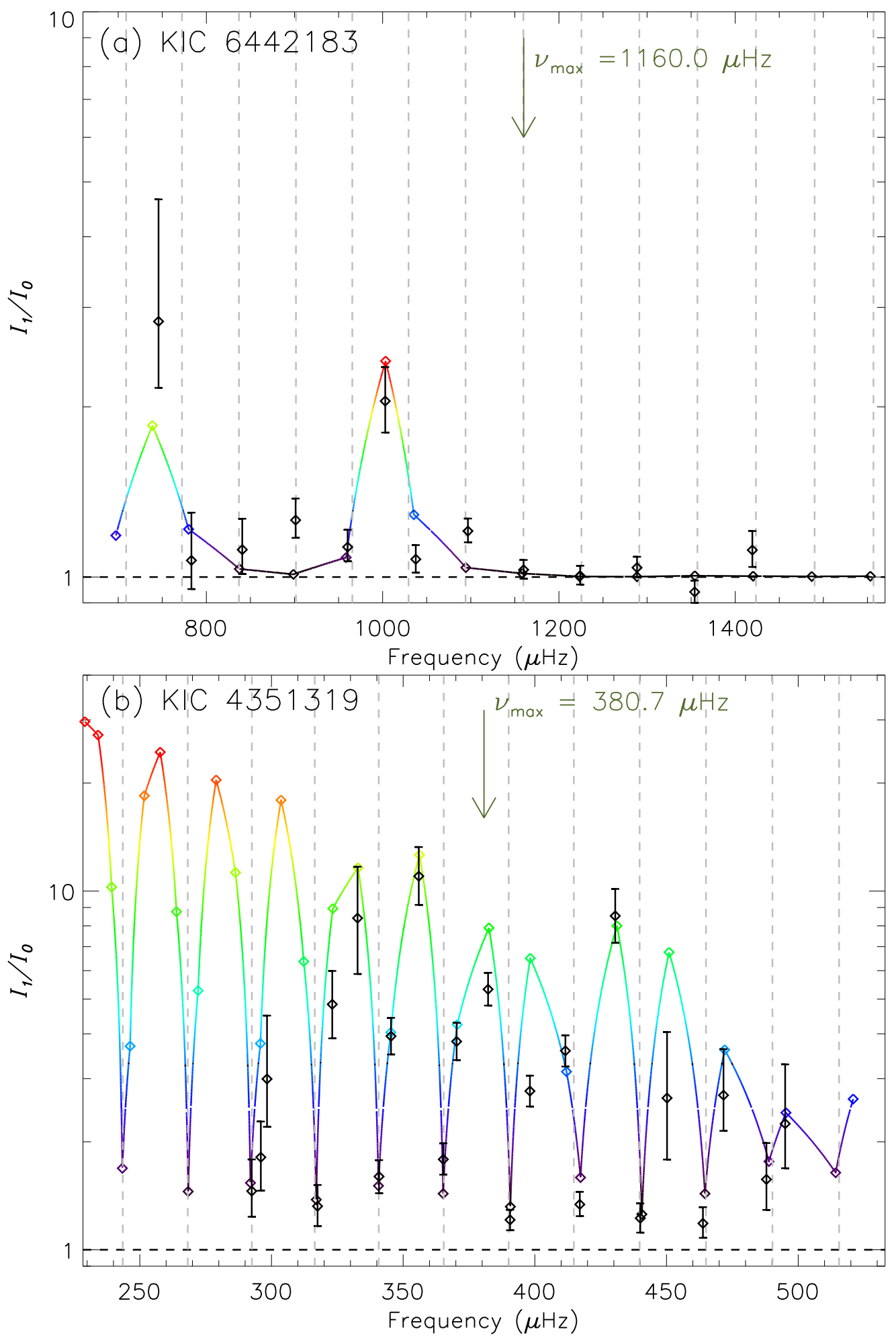

Figure 2. Measured inertia ratio between $\ell=1$ and $\ell=0$ modes against frequency (black diamonds) for KIC 4351319 (a) and KIC 6442183 (b). Theoretical inertia ratio are superimposed (colored points connected by lines). Dashed line indicates the lower limit of inertia ratio (pure p modes). The higher is the inertia, the stronger the mixed character of the mode. 

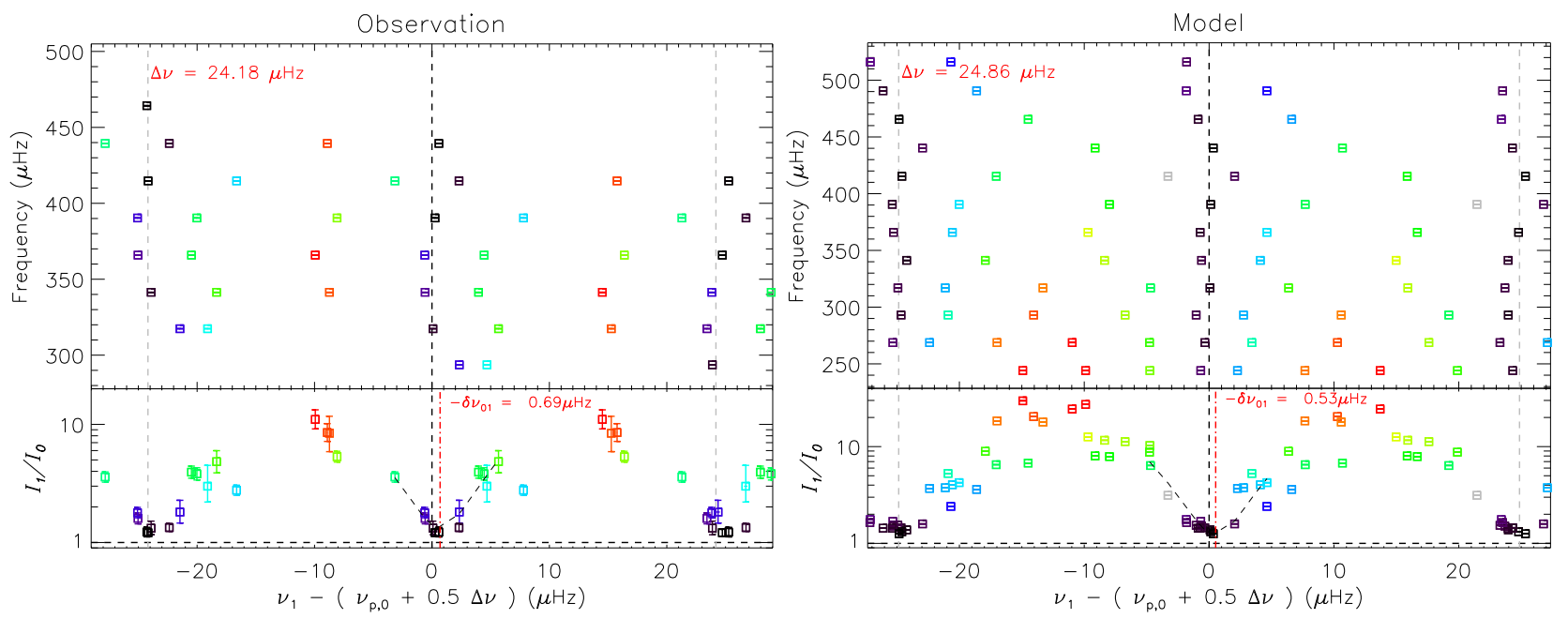

Figure 3. Frequency-échelle (top) and collapsed frequency-échelle diagram for KIC 4351319 for observations (left) and for model B (right). The minima of inertia are spaced by $\Delta \nu$. The distance to 0 in the abscissa measures $\delta \nu_{01}$.

measure the period spacing in evolved stars, with a precision of better than $1 \%$. The higher the g mode density, the smaller the uncertainty. For KIC 4351319 we found $\Delta \Pi_{1}=97.2 \mathrm{sec}$. This period spacing is compatible with that derived by other methods such as the asymptotic approach by Mosser et al. (2012b), which applied to our data gives $\Delta \Pi_{1}=96.5 \mathrm{sec}$. We also computed the period spacing of the model 1 of Di Mauro et al. (2011) and found a value of $97.4 \mathrm{sec}$, which is compatible with the value of $97.8 \mathrm{sec}$ found by integrating the Brunt Väisälä frequency (e.g. Christensen-Dalsgaard 2012). Relative uncertainties are $\simeq 0.1 \%$ here.

\section{DISCUSSION AND CONCLUSION}

In this Letter, we presented a method to measure mode-inertia ratios, a quantity which until now, was only derived from modelling. This diagnostic is based on the detection and precise measurement of mixed-mode amplitudes and linewidths. Using Kepler data, we demonstrated that it is possible to measure precisely the mode inertias of subgiants and early red giants. These can be used to determine the period spacing and the coupling between the $p$ and $g$ cavities of mixed modes. Those results were validated by comparing mode-inertia ratios derived from the observations and from stellar and pulsational modelling of KIC 4351319 and KIC 6442183.

To go a step further, and to better evaluate which constraints inertias may provide, it is useful to adopt an asymptotic approach. For this, we follow the work of Goupil et al. (2013) based on the asymptotic formalism of Shibahashi (1979) for mixed modes. This allows us to write

$$
\frac{I_{1}}{I_{0}} \approx\left\{1+\frac{1}{4} q^{2}\left(\frac{\kappa_{g}}{\kappa_{p}}\right)\left(\frac{\cos \kappa_{p}}{\cos \kappa_{g}}\right)^{2}\right\},
$$

where $q$ measures the coupling between the $p$ - and $g$ mode cavities, as introduced by Mosser et al. (2012b), and

$$
\kappa_{p} \approx \frac{\pi \nu}{\Delta \nu}, \quad \text { and } \quad \kappa_{g} \approx \frac{\pi}{\Delta \Pi_{1} \nu}-\frac{\pi}{2}
$$

where $\nu$ is the frequency. In Equation 10 and Equation 11. the period of the oscillation in the ratio $I_{1} / I_{0}$ is given by $\kappa_{g}$ (thus by the period spacing $\Delta \Pi_{1}$ ) appearing as the argument of the cosine function in Equation 10. This is due to the fact that the term $\cos \left(\kappa_{p}\right)$ is almost constant over the considered frequency range.

Moreover, the minimum value of the inertia ratio is determined by the ratio $\kappa_{g} / \kappa_{p}$ in Equation 10, which depends on the inverse of the squared frequency $\left(1 / \nu^{2}\right)$. We also note that the right-hand side of this equation is directly proportional to $q^{2}$, which is related to the strength of the coupling and characterizes the evanescent region. Therefore, Equation [10 and Equation[11 confim that the ratio $I_{1} / I_{0}$ can be used as a tool to derive the period spacing and also to constrain the evanescent region between the $p$ and $g$ cavities. This is in agreement with the results of Sect. 4.1.1.

The ratio $I_{1} / I_{0}$ provides an alternative to using mixed mode frequencies and gives essentially the same constraints (see Equation 9 of Mosser et al. 2012b). However, we note that the relationship of $I_{1} / I_{0}$ as a function of frequency to (i) the period spacing $\left(\Delta \Pi_{1}\right)$ and (ii) the properties of the inner-most layers characterised by $q$ is different than Equation 9 of Mosser et al. (2012b). Therefore, the two observational constraints are complementary, potentially providing stronger constraints on the stellar structure. Further investigations are required to evaluate the advantages of observed inertia as constrains on the stellar structure.

We stress that inertia ratios are sensitive to nonadiabacitic effects, not accounted by Ballot et al. (2011) when computing visibilities (Toutain \& Gouttebroze 1993). Mosser et al. (2012a) noted that height ratios can differ by $10 \%$ from adiabatically computed values, possibly biasing inertia ratios by $5 \%$.

Our approach requires well-resolved mixed modes and well-identifed mode rotationnal splittings, which can only be achieved with long observations. This limitation is severe for the more evolved stars because their modes have the longest lifetimes. The observations used here, spanning more than three years, resolve mixed modes down to $\nu_{\max } \simeq 120 \mu \mathrm{Hz}$. We expect to push this limit even further with the latest Kepler observations (reaching four years). 

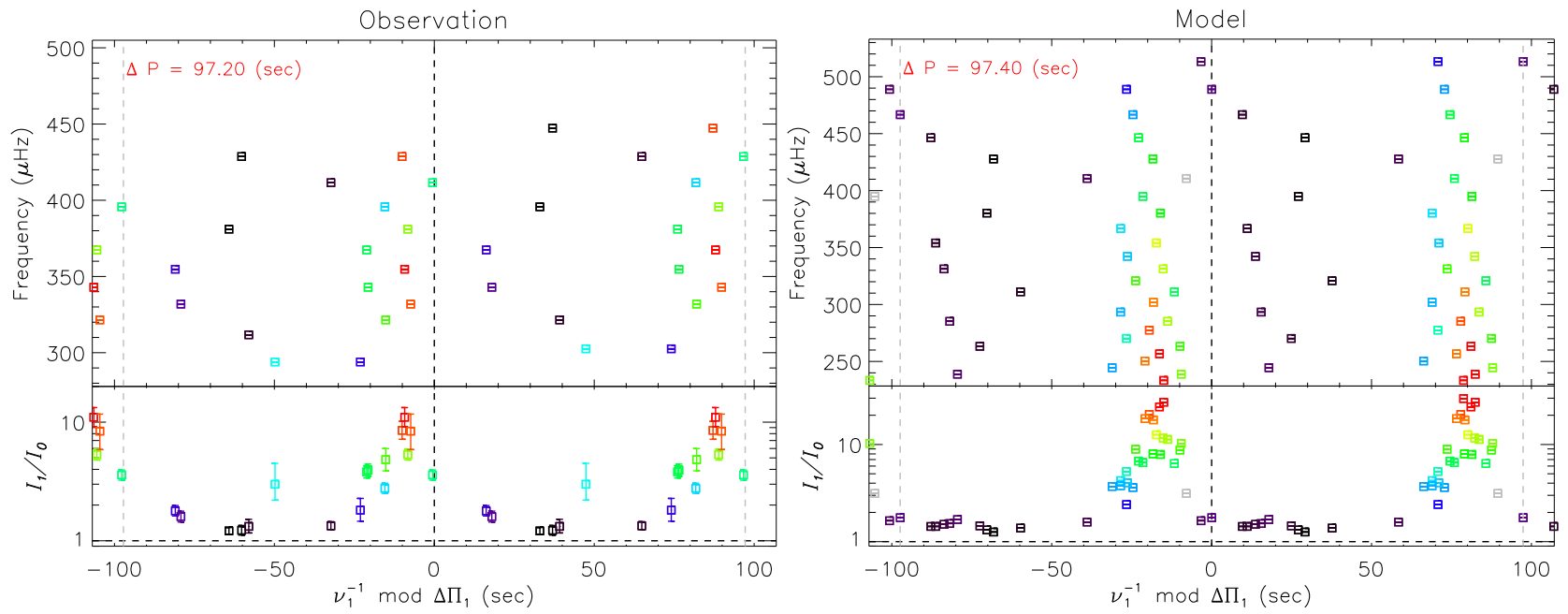

Figure 4. Period-échelle (top) and collapsed period-échelle diagram for KIC 4351319 for observations (left) and for model B (right). The maxima of inertia are spaced by $\Delta \Pi_{1}$.

Acknowledgments. "KB, BM, MJG, and RS acknowledge the ANR (Agence Nationale de la Recherche, France) program IDEE ( $\left.n^{\circ} A N R-12-B S 05-0008\right)$ "Interaction Des Étoiles et des Exoplanètes" as well as financial support from "Programme National de Physique Stellaire" (PNPS) of CNRS/INSU, France". RAG acknowledge the support of the European Community's Seventh Framework Programme (FP7/2007-2013) under grant agreement no. 269194 (IRSES/ASK).

\section{REFERENCES}

Aizenman, M., Smeyers, P., \& Weigert, A. 1977, A\&A, 58, 41 Appourchaux, T., et al. 2008, A\&A, 488, 705

-. 2012, A\&A, 543, A54

Baglin, A., Auvergne, M., Barge, P., Deleuil, M., Catala, C., Michel, E., Weiss, W., \& COROT Team. 2006a, in ESA Special Publication, Vol. 1306, ESA Special Publication, ed.

M. Fridlund, A. Baglin, J. Lochard, \& L. Conroy, 33

Baglin, A., et al. 2006b, in COSPAR Meeting, Vol. 36, 36th COSPAR Scientific Assembly, 3749

Ballot, J., Barban, C., \& van't Veer-Menneret, C. 2011, A\&A, 531, A124

Baudin, F., Samadi, R., Goupil, M.-J., Appourchaux, T., Barban, C., Boumier, P., Chaplin, W. J., \& Gouttebroze, P. 2005, A\&A, 433, 349

Beck, P. G., et al. 2011, Science, 332, 205

Bedding, T. R., et al. 2010, ApJ, 713, L176

-. 2011, Nature, 471, 608

Belkacem, K., Samadi, R., Goupil, M. J., Dupret, M. A., Brun, A. S., \& Baudin, F. 2009, A\&A, 494, 191

Belkacem, K., Samadi, R., Goupil, M. J., Kupka, F., \& Baudin, F. 2006, A\&A, 460, 183

Benomar, O., Appourchaux, T., \& Baudin, F. 2009, A\&A, 506, 15

Benomar, O., et al. 2013, ApJ, 767, 158

Borucki, W. J., et al. 2010, Science, 327, 977

Chaplin, W. J., Elsworth, Y., Isaak, G. R., Lines, R., McLeod, C. P., Miller, B. A., \& New, R. 1998, MNRAS, 298, L7
Chaplin, W. J., Houdek, G., Elsworth, Y., Gough, D. O., Isaak, G. R., \& New, R. 2005, MNRAS, 360, 859

Christensen-Dalsgaard, J. 2004, Sol. Phys., 220, 137

-. 2008a, Ap\&SS, 316, 113

-. 2008b, Ap\&SS, 316, 13

—. 2012, Astronomische Nachrichten, 333, 914

Corsaro, E., et al. 2012, ApJ, 757, 190

Di Mauro, M. P., et al. 2011, MNRAS, 415, 3783

Dupret, M.-A., et al. 2009, A\&A, 506, 57

Dziembowski, W. A. 1971, Acta Astron., 21, 289

Dziembowski, W. A., Gough, D. O., Houdek, G., \& Sienkiewicz, R. 2001, MNRAS, 328, 601

García, R. A., et al. 2011, MNRAS, 414, L6

Gizon, L., et al. 2013, Proceedings of the National Academy of Science, 110, 13267

Goldreich, P., Murray, N., \& Kumar, P. 1994, ApJ, 424, 466

Goupil, M. J., Mosser, B., Marques, J. P., Ouazzani, R. M., Belkacem, K., Lebreton, Y., \& Samadi, R. 2013, A\&A, 549, A75

Handberg, R., \& Campante, T. L. 2011, A\&A, 527, A56

Libbrecht, K. G. 1988, ApJ, 334, 510

Metcalfe, T. S., et al. 2012, ApJ, 748, L10

Michel, E., Samadi, R., Baudin, F., Barban, C., Appourchaux, T., \& Auvergne, M. 2009, A\&A, 495, 979

Michel, E., et al. 2008, Science, 322, 558

Molenda-Zakowicz, J., et al. 2013, ArXiv e-prints

Montalbán, J., Miglio, A., Noels, A., Scuflaire, R., \& Ventura, P. 2010, Astronomische Nachrichten, 331, 1010

Mosser, B., et al. 2011a, A\&A, 532, A86

-. 2011b, A\&A, 525, L9

-. 2012a, A\&A, 537, A30

-. 2012b, A\&A, 540, A143

Osaki, J. 1975, PASJ, 27, 237

Roxburgh, I. W. 2009, A\&A, 493, 185

Samadi, R., Belkacem, K., Goupil, M. J., Dupret, M.-A., \&

Kupka, F. 2008, A\&A, 489, 291

Scuflaire, R. 1974, A\&A, 36, 107

Shibahashi, H. 1979, PASJ, 31, 87

Toutain, T., \& Gouttebroze, P. 1993, A\&A, 268, 309 